\title{
Pancreatic Intraductal Papillary-Mucinous Neoplasm with Low Grade Dysplasia
}

National Cancer Institute

\section{Source}

National Cancer Institute. Pancreatic Intraductal Papillary-Mucinous Neoplasm with Low

Grade Dysplasia. NCI Thesaurus. Code C41249.

A non-invasive pancreatic intraductal papillary mucinous neoplasm characterized by the presence of neoplastic epithelial cells that form a single layer and are well polarized. The neoplastic cells exhibit small and uniform nuclei, mild pleomorphism, and rare mitotic figures. 